\title{
Mast cell IL-4 expression is regulated by Ikaros and influences encephalitogenic Th1 responses in EAE
}

\author{
Gregory D. Gregory, ${ }^{1,2}$ Shveta S. Raju, ${ }^{3}$ Susan Winandy, ${ }^{1}$ and Melissa A. Brown ${ }^{1}$ \\ ${ }^{1}$ Department of Microbiology-Immunology, Northwestern University Feinberg School of Medicine, Chicago, Illinois, USA. ${ }^{2}$ Graduate Program in Immunology \\ and Molecular Pathogenesis and ${ }^{3}$ Department of Pathology and Laboratory Medicine, Emory University School of Medicine, Atlanta, Georgia, USA.
}

\begin{abstract}
When exposed to a pathogen, a naive $\mathrm{CD} 4^{+} \mathrm{T}$ cell is forced to make a cell fate decision that leads to a polarized population of Th1 IFN- $\gamma$ - or Th2 IL-4- producing cells. Although IL-4 has traditionally been considered a factor that promotes Th2 cell differentiation, recent evidence has demonstrated that the site and timing of IL-4 expression in an immune response determines its ultimate effects on $\mathrm{CD}^{+} \mathrm{T}$ cell fate. Using a mast cell (MC) reconstitution model, we demonstrate that MC-derived IL-4 promoted Th1 responses in vivo. Furthermore, MCs from genetically disparate mouse strains varied in their potential for IL-4 expression. Independent of the activation mode, MCs from Th1-prone C57BL/6 mice exhibited a more robust $\mathrm{Il} 4$ response than did the Th2prone strain Balb/c. The hierarchy of IL-4 expression potential was directly associated with the degree of basal chromatin accessibility at cis-regulatory elements conserved noncoding sequence- 1 and $V_{A}$ enhancer within the Th2 locus. GATA1/2 and Ikaros, factors with opposing roles in chromatin remodeling, acted at these sites. We propose that GATA and Ikaros proteins coordinately fine-tune accessibility at the Il4 locus during development to variably regulate IL-4 expression. These events likely contribute to the genetically determined heterogeneity in Th1 responses that underlie susceptibility to many diseases.
\end{abstract}

\section{Introduction}

IL-4 is a multifunctional cytokine that regulates both innate and adaptive immunity (1). Cells of several lineages produce IL-4, including CD4 and CD8 T cells, NKT cells, eosinophils, mast cells (MCs), and basophils. It is likely that expression by each cell type contributes to distinct as well as overlapping physiologic responses. IL-4 is perhaps best known for its role in promoting Th2 cell differentiation and limiting Th1 responses; however, recent evidence shows that IL-4 can also direct Th1 responses (2). IL-4 can act on a wide variety of other target cells including B cells, macrophages, DCs, and MCs as well as nonimmune cells such as epithelial cells and goblet cells (3-6). This broad range of effects necessitates strict controls on the expression of this cytokine. Inappropriate expression is associated with allergic disease, autoimmunity, and failure to clear certain infections (7).

Most information regarding mechanisms of IL-4 gene (Il4) regulation comes from studies in Th cells and MCs. In addition to acute signals that elicit active Il4 transcription in both cell types, a crucial determinant of IL-4 expression is locus accessibility acquired during development (8). Il4 is closely linked to the genes encoding IL-5 (Il5) and IL-13 (Il13). These genes are clustered within an approximately $200-\mathrm{kb}$ region, termed the Th2 locus, on chromosome 11 in mice and chromosome 5 in humans. During Th cell differentiation, the Th2 locus undergoes a series of chromatin modifications that confer the relative potential for transcription

Nonstandard abbreviations used: B6, C57BL/6; BMMC, bone marrow-derived MC; CD40L, CD40 ligand; ChIP, chromatin immunoprecipitation; CNS-1, conserved noncoding sequence-1; FCERI, high affinity IgE receptor; HDAC, histone deacetylase; HS, hypersensitive site; IE, intronic enhancer; MC, mast cell; MOG, myelin oligodendrocyte glycoprotein peptide; NuRD, nucleosome remodeler and deacetylase; $\mathrm{V}_{\mathrm{A}} \mathrm{E}$, $\mathrm{V}_{\mathrm{A}}$ enhancer; W/W $\mathrm{W}^{\mathrm{v}}$, WBB6-F1/J-Kit ${ }^{\mathrm{W}} / \mathrm{Kit}^{\mathrm{W}}{ }^{\mathrm{W}}$.

Conflict of interest: The authors have declared that no conflict of interest exists. Citation for this article: J. Clin. Invest. 116:1327-1336 (2006). doi:10.1172/JCI27227. from Il4 (8). DNAse I hypersensitive site (HS) analyses and crossspecies sequence comparisons have allowed the identification of a number of cis-regulatory elements implicated in controlling Il4 expression. Included among these are regions between Il13 and Il4 (conserved noncoding sequence-1 [CNS-1]), within the Il4 promoter (HS I) and second intron (intronic enhancer [IE]), and distal to Il4 (HS IV, V, and $\mathrm{V}_{\mathrm{A}}$ ) (9-13). In addition, a locus control region is located upstream of $I l 13$ at the $3^{\prime}$ end of Rad50 (14). The histones associated with these sites become hyperacetylated in developing Th2 cells, correlating with the potential to express IL-4, whereas the Th2 locus is hypoacetylated and relegated to pericentromeric heterochromatin in Th1 cells (15-17). Resting bone marrow-derived MCs (BMMCs) express most of the same HS sites as Th2 cells within the Il4 locus. Although most currently defined sites have enhancing activity, Rao and colleagues have defined HS IV as a conserved silencer of Il4 in both T cells and MCs (9).

The Il4 regulation paradigm is based primarily on results of experiments utilizing $\mathrm{T}$ cells and MCs from only 2 common strains of mice: C57BL/6 (B6) and Balb/c. These strains have been useful in studying genetically based disease susceptibility due to their disparate immune responses to some antigens, events perhaps most clearly manifested in Leishmania major infection (18). B6 mice clear the infection due to a Th1-dominated response, whereas Balb/c mice fail to clear the parasite despite a strong but nonprotective Th2-skewed response. Most studies have focused on possible intrinsic differences in the response of B6- versus $\mathrm{Balb} / \mathrm{c}$-derived $\mathrm{T}$ cells, such as instability in expression of the IL-12 receptor $\beta 2$ subunit in Balb/c T cells (19). We considered the possibility that strain-specific differences in bystander cell cytokine expression exist that modulate the Th1/Th2 balance. A genetically determined difference in the kinetics or level of IL-4 expression by MCs, for example, could directly alter the character of the $\mathrm{T}$ cell response or act indirectly through effects on DC 

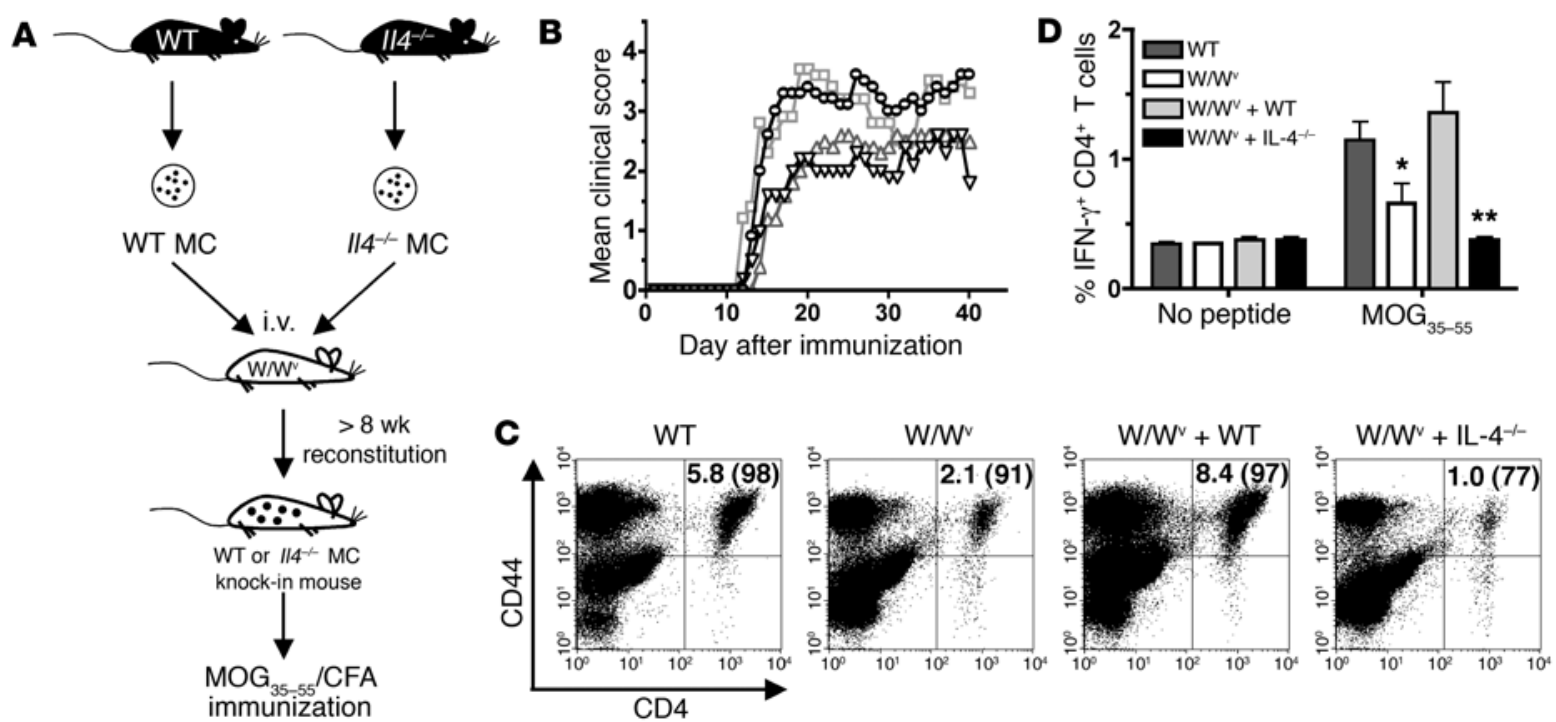

Figure 1

MC-derived IL-4 enhances the severity of EAE by promoting the generation of MOG $_{35-55}$-specific IFN- $\gamma$-expressing Th1 cells. (A) Diagram of the $M C$ reconstitution protocol. MCs were generated by in vitro differentiation and transferred i.v. to MC-deficient W/Wv mice. (B) Mice were immunized with $\mathrm{MOG}_{35-55}$ peptide to induce $\mathrm{EAE}$ and monitored daily for clinical signs of paralysis. Mean clinical scores were determined daily $(n=5$ per

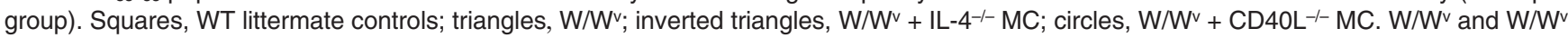
$+\mathrm{IL}-4^{-1-} \mathrm{MC}$ mice exhibited significantly reduced EAE severity compared with WT littermate controls $(P<0.01)$. (C) WT, W/Wv, and W/Wv mice reconstituted with $\mathrm{B} 6$ or IL-4-/- BMMCs were perfused at 11 days after immunization, and flow cytometric analysis of CNS-infiltrating CD44hi CD4 T cells was performed. Numbers in quadrants represent the percentage of CD4 T cells; numbers in parenthesis indicate the percentage of CD4 $\mathrm{T}$ cells that are CD44hi. (D) Antigen-specific CD4+ $\mathrm{T}$ cell responses were measured by intracellular cytokine staining for IFN- $\gamma$ after a brief in vitro restimulation of splenocytes with $\mathrm{MOG}_{35-55}$ peptide. ${ }^{*} P<0.05$ versus $\mathrm{WT}$; ${ }^{* \star} P<0.01$ versus $\mathrm{W} / \mathrm{W}^{\mathrm{v}}+\mathrm{WT}$. For reconstitution in $\mathbf{C}$ and $\mathbf{D}$, B6-derived BMMCs were used as controls for IL-4 ${ }^{--}$BMMCs, whereas WBB6-F1/J-Kit $/ \mathrm{Kit}^{+} \mathrm{WT}$ littermates were used as controls for W/W $\mathrm{Wice}^{\mathrm{V}}$.

maturation. In this report, we demonstrate that MC IL-4 was critical to the generation of myelin-specific Th1 cells and contributed to severe EAE, a murine model of MS. Furthermore, we show that there were strain-specific differences in murine MC Il4 expression potential that may influence the heterogeneity of Th1 versus Th2 responses. Variability in the expression of Il4 occurred irrespective of the mode of activation and correlated with distinct patterns of basal $\mathrm{H} 3$ and $\mathrm{H} 4$ histone acetylation at several sites within the Th2 locus, including CNS-1 and $V_{A}$ enhancer $\left(V_{A} E\right)$ elements. GATA1, GATA2, and Ikaros, DNA-binding proteins with established roles in chromatin remodeling, associated with CNS-1 and $\mathrm{V}_{\mathrm{A}} \mathrm{E}$ in vivo. We provide direct evidence that Ikaros has a suppressive role in MC Il4 transcription, as Ikaros ${ }^{\text {null }} \mathrm{MCs}$ had elevated IL-4 mRNA expression and chromatin accessibility across the Th2 locus. Our data is consistent with a model whereby the interplay of GATA and Ikaros proteins determines the variable potential for MC Il4 expression, which subsequently exerts a direct effect on Th1/Th2 responses.

\section{Results}

$M C$-derived IL-4 influences EAE severity by enhancing encephalitogenicity of Th1 cells. We hypothesized that MCs, by virtue of their ability to express cytokines that modulate $\mathrm{T}$ cell responses, have a role in adaptive immune responses (20). Consistent with this idea are results of previous studies showing that MC-derived TNF- $\alpha$ regulates in vitro T cell reactivity (21) and that MCs are essential for optimal generation of encephalitogenic Th1 cells during EAE (22). Although IL-4 is best known as a Th2-polarizing cytokine, IL-4 can also have distinct effects on Th1 responses $(2,23,24)$. Thus it was of interest to investigate whether MC IL-4 expression could modulate the quality of a Th1-mediated response.

Using a MC knock-in model in which MC-deficient WBB6-F1/ $\mathrm{J}-\mathrm{Kit}^{\mathrm{W}}-\mathrm{Kit}^{\mathrm{Wv}}\left(\mathrm{W} / \mathrm{W}^{v}\right)$ mice were selectively reconstituted with either WT or IL-4 $4^{-/}$BMMCs (referred to as W/Wv $+W^{\mathrm{V} T}$ and $\mathrm{W} / \mathrm{W}^{\mathrm{v}}+$ IL-4 ${ }^{-/}$, respectively; Figure $1 \mathrm{~A}$ ), we assessed the specific effects of MC IL-4 on myelin oligodendrocyte glycoprotein peptide-induced (MOG $35-55$-induced) EAE. As previously reported, $\mathrm{W} / \mathrm{W}^{\mathrm{v}}$ mice exhibited a significantly reduced overall mean clinical score compared with WT littermate controls $(P<0.01$; Figure 1B). Reconstitution of $\mathrm{W} / \mathrm{W}^{v}$ mice with MCs deficient in CD40 ligand (CD40L), a cell surface costimulatory molecule expressed on several cell types, restores disease to WT levels and served as a positive control in this experiment (25). In contrast, mice reconstituted with IL-4-/MCs failed to display severe disease.

Development of EAE and MS is dependent upon trafficking of activated, myelin-specific T cells to the CNS. Consistent with reduced disease severity, reduced CNS infiltration of CD4 T cells was observed in $\mathrm{W} / \mathrm{W}^{\mathrm{v}}$ and $\mathrm{W} / \mathrm{W}^{\mathrm{v}}+\mathrm{IL}^{-4^{-/}}$mice compared with WT and W/Wv + WT mice (Figure $1 \mathrm{C}$ ). Furthermore, appreciably fewer $\mathrm{T}$ cells entering the $\mathrm{CNS}$ of $\mathrm{W} / \mathrm{W}^{\mathrm{v}}$ and $\mathrm{W} / \mathrm{W}^{\mathrm{v}}+\mathrm{IL}-4^{-/-}$mice were activated, as measured by high CD44 surface expression. Together, these results suggest that MC IL-4 promotes encephalitogenic Th1 responses.

$\mathrm{MOG}_{35-55}$-specific $\mathrm{T}$ cell responses were also evaluated in WT and reconstituted mice. As shown in Figure $1 \mathrm{D}, \mathrm{W} / \mathrm{W}^{\mathrm{v}} \mathrm{T}$ cell responses were reduced relative to WT as previously reported (22). While WT BMMCs restored the frequency of $\mathrm{MOG}_{35-55}$-specific IFN- $\gamma$-producing CD4 T cells, IL-4-/- BMMCs did not $(P<0.01)$. 

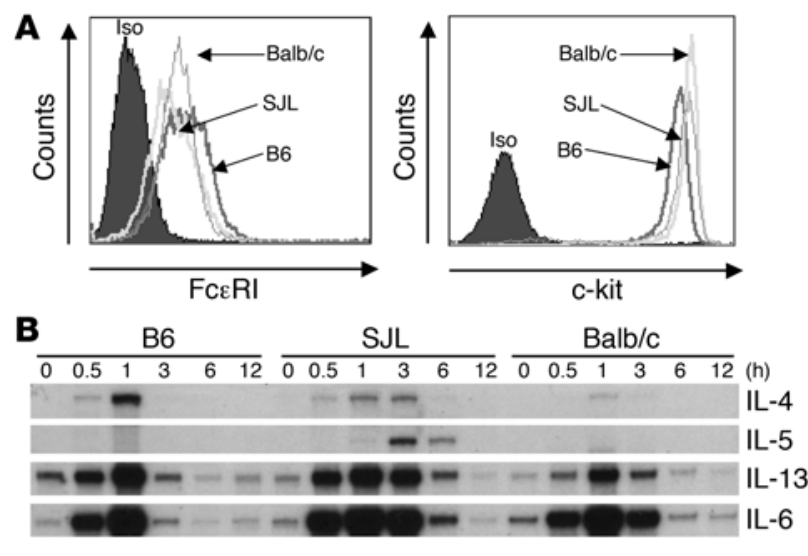

$000000000000000 \mathrm{GAPDH}$
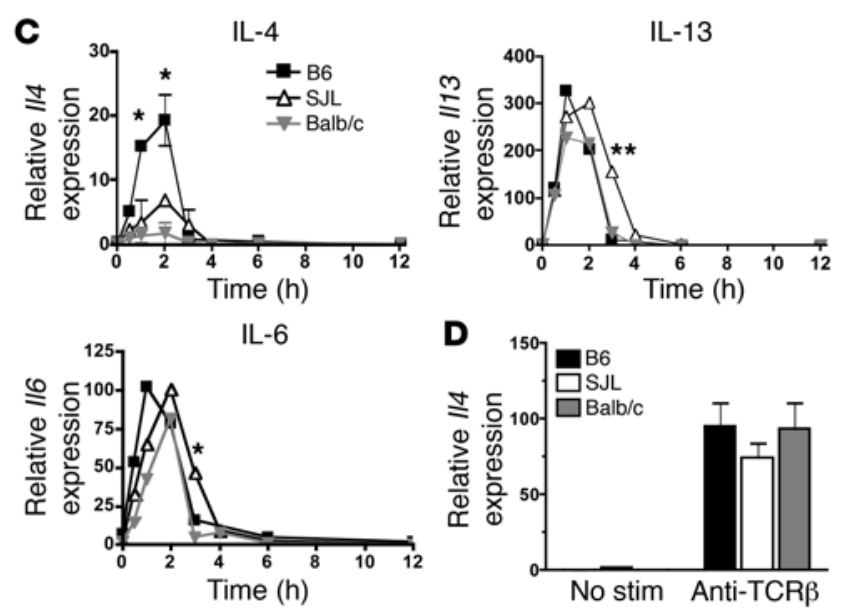

Similar results were obtained in experiments using immunization with OVA $323-339$, a nonencephalitogenic peptide (data not shown).

MCs, but not Th2 cells, exhibit strain-specific differences in Il4 expression. The demonstration that MC IL-4 enhanced Th1 responses raised the possibility that the propensity for Th heterogeneity in various strains of mice might be due to intrinsic differences in the magnitude of MC IL-4 expression. For example, MCs from Th1-prone mice may have relatively higher IL-4 expression compared with a Th2-dominated strain. MCs from the B6 and Balb/c strains were chosen to examine potential strain-specific differences in MC Il4 expression due to their dichotomous responses to L. major infection (18). The SJL strain was also examined as it has documented defects in T cell IL-4 expression and IgE production in vivo (26). MCs were differentiated from bone marrow by culturing in the presence of SCF and IL-3. BMMCs from all 3 strains expressed comparable levels of c-kit and the high affinity IgE receptor (FceRI) after 6-8 weeks of culture (Figure 2A). Cells were activated by cross-linking of FceRI, and IL-4 mRNA levels were measured at various times after stimulation. As shown in Figure 2, B and C, B6 BMMCs exhibited quick and relatively robust expression of Il4 compared with BMMCs from Balb/c or SJL mice $(P<0.05$ at 1 and 2 hours). SJL BMMCs showed intermediate and more prolonged expression of Il4, whereas Balb/c MCs displayed a relatively weak expression profile, demonstrating the strain-specific variations in the duration and magnitude of the IL-4 response. The expression of other genes such as $I l 5, I l 13$, and Il6 also displayed notable varia-

\section{Figure 2}

MCs exhibit strain-specific differences in I/4 expression after FceRI cross-linking. (A) Surface expression of $F c \varepsilon R I$ and c-kit on BMMCs from B6, Balb/c, and SJL mice after 6-8 weeks of culture in IL-3 and SCF. The mean fluorescent intensity of both c-kit and FceRI is essentially equivalent in all strains. Iso, isotype. (B and $\mathbf{C}$ ) Kinetics of BMMC cytokine mRNA expression after FceRI cross-linking. Ribonuclease protection assays were performed to measure cytokine transcripts from B6, Balb/c, and SJL MCs at resting state (0 hours) and at various times after stimulation by $\mathrm{Fc \varepsilon RI}$ cross-linking. Ribonuclease protection assays were visualized by autoradiography after 16 hours (II5, II6, I/13, and Gapdh) or 5 days (II4) and quantified by phosphorimaging ( $n=2-4$ per time point). mRNA levels were expressed relative to the housekeeping gene GAPDH and calculated as (cytokine intensity/ GAPDH intensity) $\times 100 .{ }^{*} P<0.05 ;{ }^{*} P<0.01$ (ANOVA followed by Bonferroni's multiple comparison test). (D) Freshly isolated naive CD4+ T cells from B6, Balb/c, and SJL mice were cultured under Th2 skewing conditions for 7 days and restimulated with plate-bound anti-TCR $\beta$. I/4 expression was measured and quantified as in $\mathbf{C}$. Two independent experiments were performed. stim, stimulation.

tions between strains. For example, appreciable $I l 5$ expression was observed only in SJL MCs.

To examine whether this difference was also evident in T cells, naive $\mathrm{CD}^{+} \mathrm{T}$ cells from the same strains were isolated, differentiated under Th2 skewing conditions, and analyzed for cytokine expression. Unlike BMMCs, Th2 cells cultured in vitro showed no significant strain-specific variation in expression of IL-4 mRNA after restimulation through the $\mathrm{T}$ cell receptor (Figure 2D).

B6-derived BMMCs express higher levels of IL-4 mRNA irrespective of activating agonist. The unique profiles of MC Il4 expression could be intrinsic to FceRI signaling; alternatively, they may reflect more global differences in basal chromatin accessibility at the Il4 locus, leading to distinct expression potentials regardless of the mode of stimulation. To distinguish between these possibilities, the expression of IL-4 mRNA was compared after TLR-induced signaling. TLR2 and TLR4 mRNA expression was confirmed in cells derived from all 3 strains (Figure 3A). Cells were then stimulated with peptidoglycan (a TLR2 agonist), LPS (a TLR4 agonist), or heat-killed Mycobacterium tuberculosis, which can signal through both TLR2 and TLR4. B6-derived MCs expressed higher levels of IL-4 mRNA in response to all agonists compared with Balb/c MCs (Figure 3, B and C). This result was consistent with the idea that B6 MCs (IL-4hi) have a greater potential for IL-4 expression irrespective of the activating stimulus.

Il4 expression potential correlates with Th2 locus accessibility. Guo et al. demonstrated that the probability of a Th2 cell producing relatively high or low levels of IL-4 directly correlates with the degree of histone acetylation, a marker of chromatin accessibility, at the Th2 cytokine locus (27). To examine whether strain-based differences in the ability of MCs to express IL-4 are reflected in distinct chromatin states that affect accessibility within the Il4 locus, histone acetylation was evaluated in resting MCs by chromatin immunoprecipitation (ChIP) using antibodies specific for acetylated histones $\mathrm{H} 3$ and $\mathrm{H} 4$. IP templates were analyzed by PCR using primers specific for previously defined regulatory elements within the Th2 locus (Figure 4A). Several differences were noted in histone $\mathrm{H} 3$ and $\mathrm{H} 4$ acetylation between strains (Figure 4, B-D). Of particular interest is the consistent and striking difference in acetylation observed at CNS- 1 and $V_{A} E$ in the IL- $4^{\text {hi }}$ B6 and IL- $4^{\text {int }}$ SJL strains relative to $\mathrm{Balb} / \mathrm{c}$. Both of these elements are necessary for 
A

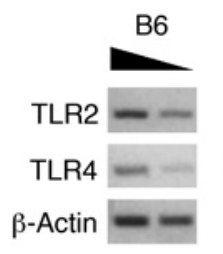

B

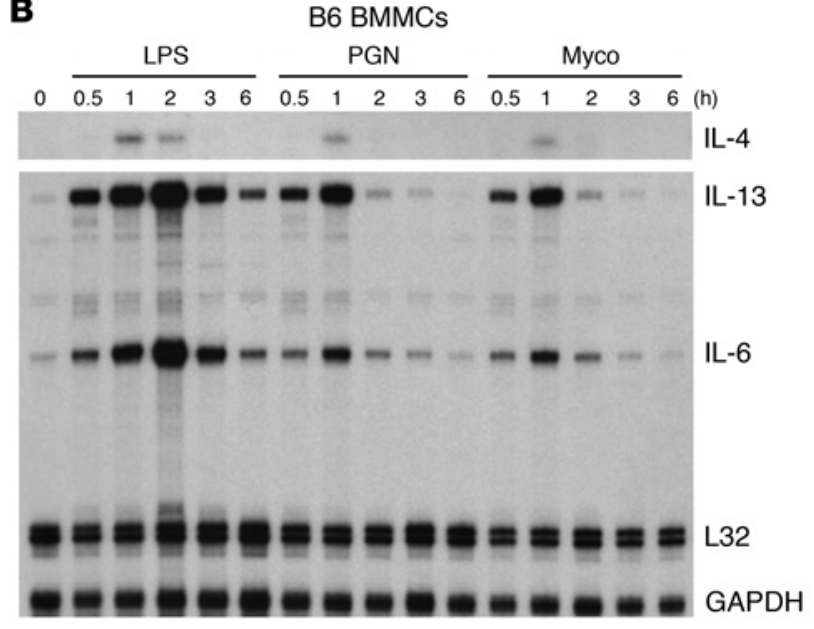

C
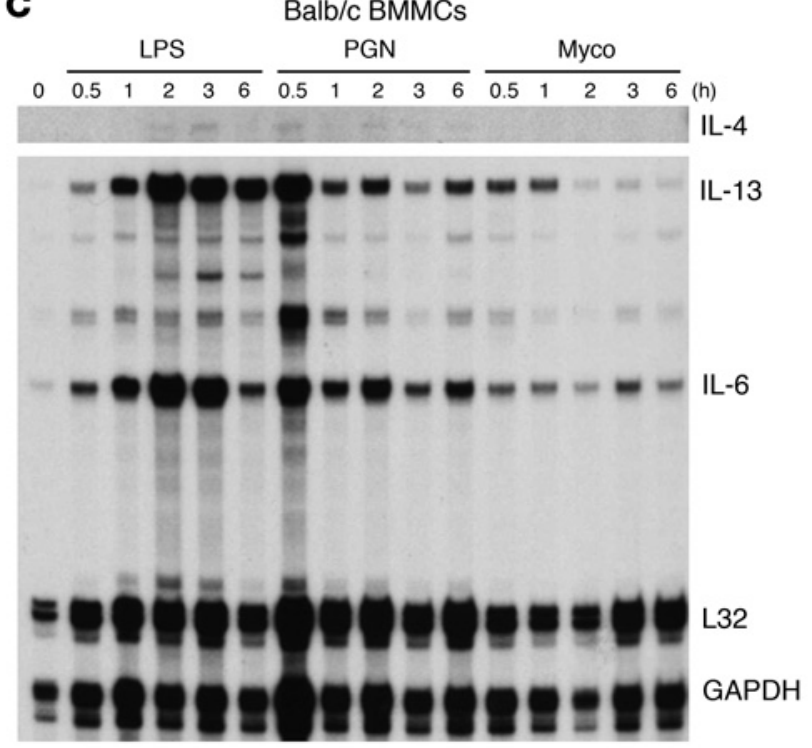

a strong transcriptional response in $T$ cells, and $V_{A} E$ is essential for optimal MC Il4 expression $(11,13,28)$. In addition, accessibility at both these sites is a key determinant in regulating the potential for high versus low IL-4 expression in T cells (27). Thus the potential for MC Il4 expression correlated with a unique pattern of chromatin accessibility at several sites within the Th2 locus.

GATA and Ikaros family members associate with CNS-1 and the $V_{A} E$ in vivo. The mechanism regulating Il4 locus accessibility in MCs as well as $\mathrm{T}$ cells is poorly understood. Our finding that the acetylation state of cis-regulatory elements CNS-1 and $\mathrm{V}_{\mathrm{A}} \mathrm{E}$ is commensurate with Il4 expression potential warranted a further investigation into the factors regulating these sites in MCs. No base pair polymorphisms were detected within these elements, indicating that strain-specific differences in $I l 4$ transcription are not due to alterations in transactivating factor binding sites within CNS-1

\section{Figure 3}

Strain-specific differences in B6 versus Balb/c //4 expression are evident after TLR stimulation. (A) TLR2 and TLR4 expression was confirmed in resting BMMCs by RT-PCR. (B and C) B6 (B) and Balb/c BMMCs (C) were stimulated with LPS, peptidoglycan (PGN), or M. tuberculosis (Myco; heat-killed) for the times indicated, and cytokine expression was determined as described in Figure 2B. Probes specific for the housekeeping genes GAPDH and ribosomal protein L32 served as controls for each sample.

or $\mathrm{V}_{\mathrm{A}} \mathrm{E}$. A number of putative GATA and Ikaros binding sites are present within both CNS-1 and $\mathrm{V}_{\mathrm{A}} \mathrm{E}$ (Figure $5 \mathrm{~A}$ ). As shown by ChIP analysis in Figure 5B, GATA1, GATA2, and Ikaros were found to associate with the CNS-1 and $V_{A} E$ elements. Binding to $V_{A} E$ was generally more variable compared with CNS-1. The demonstration that GATA and Ikaros associated with these sites in nonstimulated MCs suggests that they are involved in regulating the Th2 locus.

Ikaros regulates IL-4 expression in MCs. We have previously shown that GATA1 and GATA2, like GATA3 in Th2 cells, are positive regulators of Il4 expression in MCs. Association of these factors with the IE is critical for intron acetylation and subsequent transcription (29-31). In contrast to the sole positive actions of GATA family members, Ikaros can act as either an activator or a suppressor of transcription, activating expression of CD8 $\alpha$ in T cells and suppressing $\lambda 5$ expression in B cells $(32,33)$. To investigate the effect of Ikaros on Il4 expression, BMMCs were derived from $(\mathrm{B} 6 \times 129)$ F1 mice containing a targeted deletion of Ikaros (Ikaros $\left.{ }^{\text {null }}\right)$ and their wild-type littermates (Ikaros ${ }^{+/+}$) using standard differentiation culture conditions. RT-PCR assays revealed that $\mathrm{Ikaros}^{+/+} \mathrm{MCs}$ expressed a variety of Ikaros isoforms and confirmed the absence of Ikaros expression in Ikaros ${ }^{\text {null }}$ MCs (Figure 6A). Like Ikaros ${ }^{+/+}$, Ikaros $^{\text {null }} \mathrm{MCs}$ were c-kithi and FcERI ${ }^{+}$and contained the characteristic metachromatic granules (Figure 6B and data not shown), indicating that there were no defects in the ability to generate MCs from bone marrow. Although basal expression of FceRI was consistently lower in Ikaros ${ }^{\text {null }}$ cells, treatment with monomeric IgE resulted in identical increases in receptor expression, confirming that downstream FceRI-signaling pathways were intact (Figure 6B) (34). Ikaros ${ }^{\text {null }} \mathrm{BMMCs}$ demonstrated elevated and more prolonged expression of Il4 in response to FceRI cross-linking compared with Ikaros $^{+/+} \mathrm{MCs}$ (Figure 6, C and D). To corroborate these findings at the protein level, activation-induced IL-4 secretion was measured in both groups (Figure 6E). IL-4 was detected in supernatants from Ikaros ${ }^{\text {null }}$ BMMCs 22 hours after FceRI cross-linking and TLR2-mediated stimulation, but not in $\mathrm{Ikaros}^{+/+}$BMMCs. TNF- $\alpha$ secretion was measured as a positive control for activation in both groups. While Ikaros ${ }^{\text {null }}$ BMMC expressed lower levels of IL-5 mRNA, expression of Il13, as well as Il6 (a cytokine gene encoded outside of the Th2 locus), was not appreciably affected. Transcription of Rad50, a gene located within the Th2 locus but expressed constitutively in most cell types and under separate control than $I l 4, I l 5$, and Il13, was not affected in resting Ikaros ${ }^{\text {null }}$ BMMCs (Figure $6 \mathrm{~F}$ ). Taken together with the evidence of in vivo Ikaros binding to Il4 regulatory elements, these data demonstrate that Ikaros is specifically involved in regulation of the Il4 locus in MCs.

Ikaros controls Il4 expression at the chromatin level. To our knowledge, a direct mechanism by which Ikaros exerts its influence on the CD $8 \alpha$ and $\lambda 5$ loci has not been described previously $(32,33)$. The increased Il4 expression observed in Ikaros ${ }^{\text {null }}$ BMMCs could be indicative of an overall suppressive effect on chromatin acces- 
A

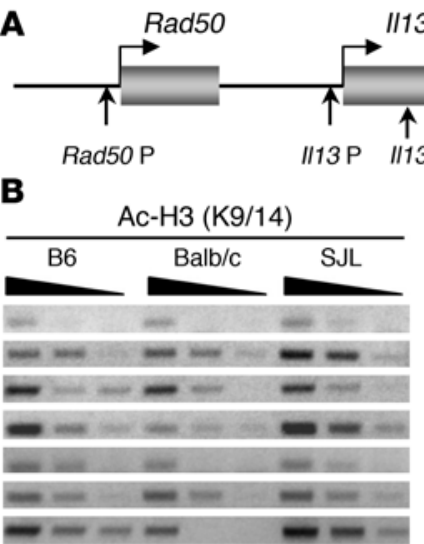

IgG control

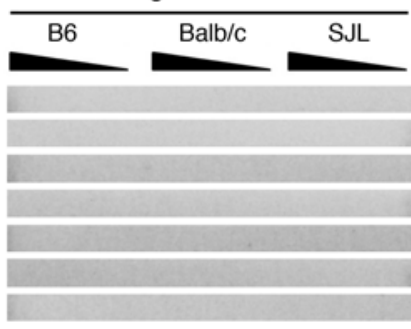

13

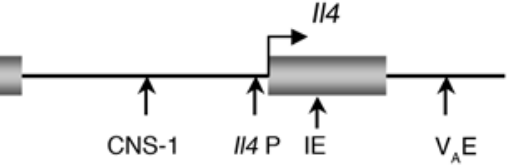

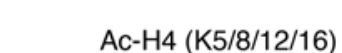

B6 $\quad$ Balb/c SJL

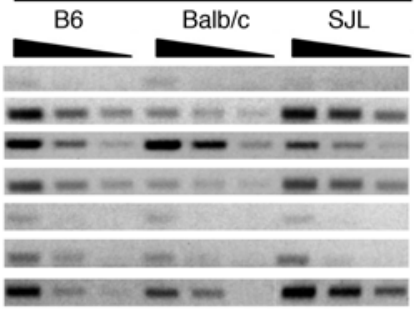

TC

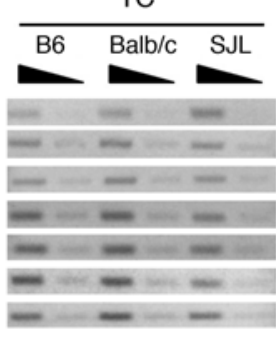

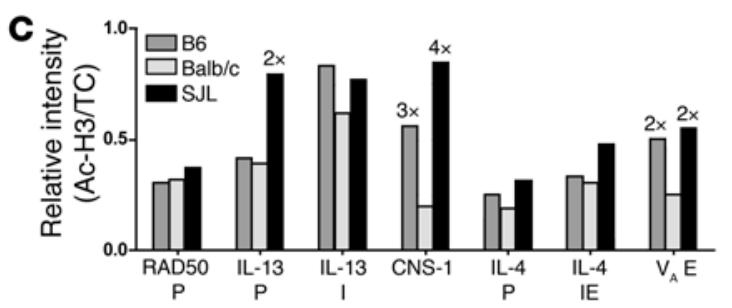

D

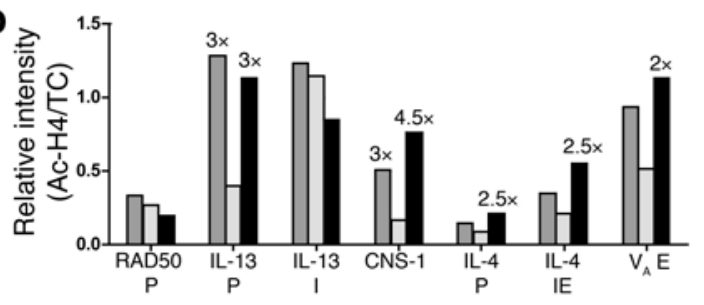

sibility at the Th2 locus. Ikaros can associate with the higher order nucleosome remodeler and deacetylase (NuRD) complex containing histone deacetylase 1 (HDAC1) and HDAC2. Thus if Ikaros is acting to recruit HDAC activity to the Il4 locus, the lack of Ikaros expression was predicted to result in higher histone acetylation levels within the Th2 locus. As expected, acetylation of histones H3 and $\mathrm{H} 4$ was increased at most of the Th2 locus regulatory elements examined in Ikaros ${ }^{\text {null }}$ BMMCs compared with Ikaros ${ }^{+/+}$BMMCs (Figure 7). Relative levels of histone acetylation at the Gapdh promoter were equivalent in both cell populations (data not shown). Thus Ikaros regulated the potential for Il4 expression in MCs by reducing chromatin accessibility at the Th2 locus.

\section{Discussion}

It is well accepted that cells of the innate immune system can profoundly influence the character of the adaptive immune response. There has been much speculation that MCs in particular can affect

\section{Figure 4}

Histone acetylation at the Th2 locus in MCs correlates with strain-dependent differences in //4 expression. (A) Diagram of the murine Th2 locus showing a subset of defined regulatory elements. $\mathrm{P}$, promoter; I, intron. (B) The relative levels of histone $\mathrm{H} 3$ and $\mathrm{H} 4$ acetylation $(\mathrm{Ac}-\mathrm{H} 3$ and $\mathrm{Ac}-\mathrm{H} 4$, respectively) at Th2 regulatory elements in resting BMMC were analyzed by ChIP assays. Antibodies specific to diacetylated histone $\mathrm{H} 3$ (lysine residues 9 and 14 [K9/14]) or tetraacetylated histone $\mathrm{H} 4(\mathrm{~K} 5 / 8 / 12 / 16)$ were used. Three-fold dilutions of IP samples were used in PCR reactions, whereas 1:10 and 1:50 dilutions were used for PCR amplification of total chromatin input (TC). Data are representative of 4 experiments using independently derived BMMC cultures. (C and D) Data was quantified using $\mathrm{NIH}$ Image - relative intensity was calculated as the ratio of IP template to total chromatin input. Relative intensities at least 2-fold greater than that of Balb/c samples are shown above bars $(2 x, 3 x$, etc. $)$.

the type and magnitude of a $\mathrm{T}$ cell response through the elaboration of immunoregulatory cytokines or expression of co-stimulatory molecules $(35,36)$. Most data supporting such a role have come from in vitro studies using long-term $\mathrm{T}$ cell and MC lines (35). An underlying hypothesis of the present investigation was that strainspecific variations in MC IL-4 expression contribute to the genetically determined heterogeneity in Th responses. Several results of our study support this hypothesis. First, we showed that there were intrinsic differences in the ability of BMMCs, but not Th2 cells, derived from the $\mathrm{B} 6, \mathrm{Balb} / \mathrm{c}$, and SJL backgrounds to express IL-4. These differences were independent of the cell activation mode and correlated with distinct patterns of basal chromatin accessibility at the Th2 locus.

How could a variable pattern of accessibility within the Th2 locus be achieved that would give rise to distinct potentials for $I l 4$ expression? A clue is provided by the observation that GATA and Ikaros, factors with apparent opposing activity at the Il4 locus, bound in vivo to regulatory elements of the Th2 locus. Notably, members of both of these families of transcription factors impact the development of specific cell lineages through their ability to modulate chromatin accessibility (37-39). In Th2 cells, GATA 3 is essential for Th2 differentiation and the events associated with Il4 locus opening (40). This factor is thought to create and maintain an open chromatin state in resting T cells (41), "poising" the locus for the interaction of inducible factors such as nuclear factor of activated T cells (NFAT) with their cognate sites upon cell activation (42-44). GATA3 enhances gene expression by increasing histone acetylation, presumably through the recruitment of $\mathrm{CBP} / \mathrm{p} 300$-like proteins containing histone acetyltransferase activity. GATA3 can also antagonize the recruitment of DNA methyltransferase- 1 and methyl CpG-binding domain protein- 2 to CNS-1 in Th2 cells, 2 factors that would otherwise mediate repression of locus accessibility $(45,46)$. In MCs, GATA1 and GATA2 appear to play an analogous role to GATA3. However, while GATA2 is absolutely required for MC differentiation, a more predominant role in regulating gene expression in mature MCs has been assigned to GATA1 $(39,47)$. Both factors associate with the Il4 IE in resting MCs and are necessary for main- 
A

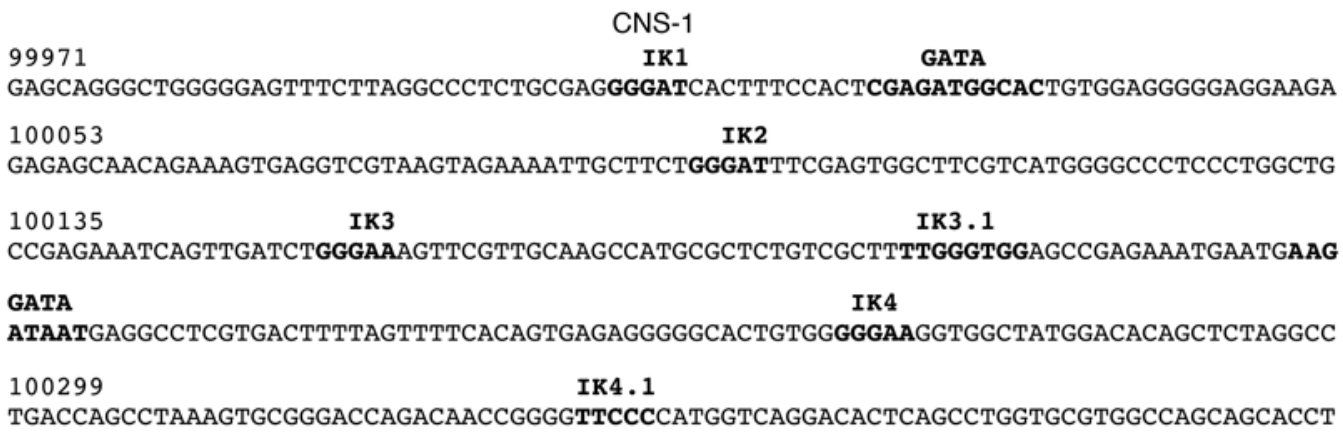

GATA

GCCACCATCTCAGAGGAGAGGAGGGAAACAGACCTGGTACCCACTGACCAGCTGCCTGCAAGGGGAAAGGCGAGCTGCAGC

100463 GATA GATA

GTAAATGTGACAGTGGATGGCTTAATTAGCCCCATCCTCCTAGCTGTCCTCAGACACCATTGTCTGACATAGGCCTAGTCCT

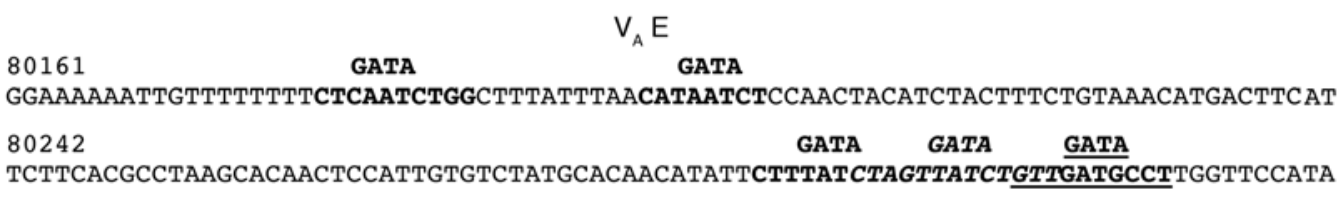

80324 GATA

TATTCATGACTGTGCAGTGCCACAATGATTGTGAATGTACAAGCATCTCTGTTGGGTTCTCAGTCCAACAGAGATATAATAT

80406

TTAATTCTTCAGAACCCTCCATACAGTTCCTGCAGTAGCTGCCCCAGCTTTCTTTGCCACTAGCAATGTTTAAGTGCCCTGG

$80488 \quad$ GATA

TGCCCTCTTGAGTATATCTTGGGGTGGGGAGATGGCTCAGTGGGGCAAGAACACTTACTAAGGTGGCATCAGGACCTGAAT

GATA

Ikaros

TCACATCTCCAGCACTCATC
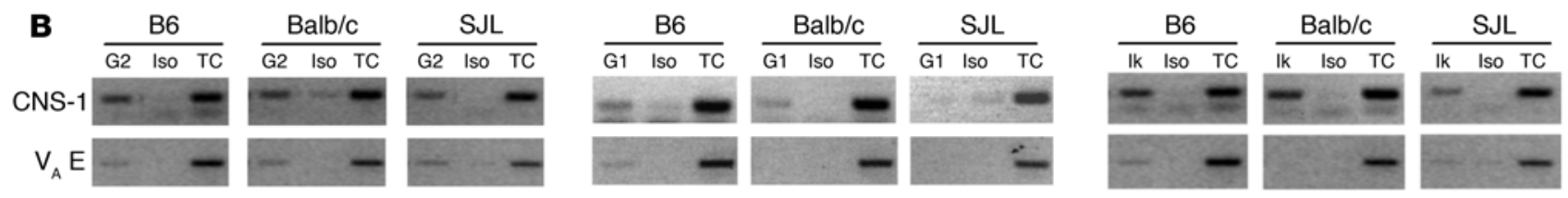

Figure 5

GATA1, GATA2, and Ikaros associate with the cis-regulatory elements CNS-1 and $V_{A} E$ in vivo. (A) Genomic sequences of murine CNS-1 and $V_{A} E$ within the Th2 locus. Sequences were analyzed using TFSEARCH (62), and putative binding motifs were identified. (B) GATA2, GATA1, and Ikaros proteins associated with CNS-1 and $\mathrm{V}_{\mathrm{A}} \mathrm{E}$. Chromatin samples isolated from resting B6, Balb/c, and SJL BMMCs were used for ChIP analyses using GATA1-, GATA2- and Ikaros-specific antibodies (G1, G2, and Ik, respectively). All samples within a particular set (antibodies, isotype, and total chromatin control) were analyzed at the same time using identical PCR conditions and electrophoresed on the same agarose gel.

taining a demethylated DNA and acetylated histone chromatin state required for transcription (29-31). Based on these previous studies, GATA family members are considered positive regulators of chromatin accessibility in both cell types.

In contrast, our data indicated that Ikaros exerts a negative influence on expression from the Th2 locus. Ikaros was first characterized as an integral component of chromatin remodeling complexes in cells of the lymphoid lineage. Mice containing a targeted deletion in Ikaros have profound defects in both B and T cell development (37). The defined molecular targets of Ikaros have been limited to only 3 genes expressed during lymphoid cell development: $\mathrm{CD} 8 \alpha$, TdT, and $\lambda 5(32,33,48)$. Our study provides what we believe to be the first direct evidence that Ikaros is also involved in Il4 regulation. Not only did Ikaros associate with cis-regulatory elements of Il4, but Ikaros ${ }^{\text {null }}$ MCs derived from $(B 6 \times 129)$ F1 mice had dysregulated Il4 expression. The increased IL-4 mRNA and protein expression as well as elevated histone acetylation at CNS-1, $\mathrm{V}_{\mathrm{A}} \mathrm{E}$, and other sites observed in Ikaros ${ }^{\text {null }} \mathrm{MCs}$ confirms that this factor exerts a suppressive influence on $\mathrm{Il} 4$.

Ikaros was initially implicated in gene repression by virtue of its association with epigenetically silenced genes in pericentromeric heterochromatin (49). In Th1 cells, CNS-1 was essential for the localization of Il4 to this nuclear compartment, an event that is associated with transcriptional blockade (16). It is hypothesized that Ikaros mediates this process through direct effects on the CNS-1 element. As IL-4 mRNA expression was still observed in Ikaros ${ }^{+/+} \mathrm{MCs}$, it is likely that Ikaros acts to modulate rather than completely suppress overall potential for transcription. The negative role of Ikaros in chromatin remodeling is also assumed to be due, in part, to its ability to form complexes with $\mathrm{NuRD}$ (50). Ikaros's repressive action on Il4 transcription in MCs may be the result of targeting of NuRDassociated HDAC activity to relevant cis-regulatory elements. 
A

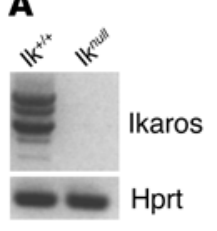

B Resting state

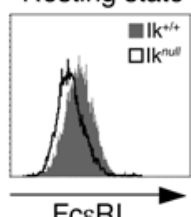

FceRI

C $\mathrm{Ik}^{+/+}$

Ik $\mathbf{k}^{\text {null }}$
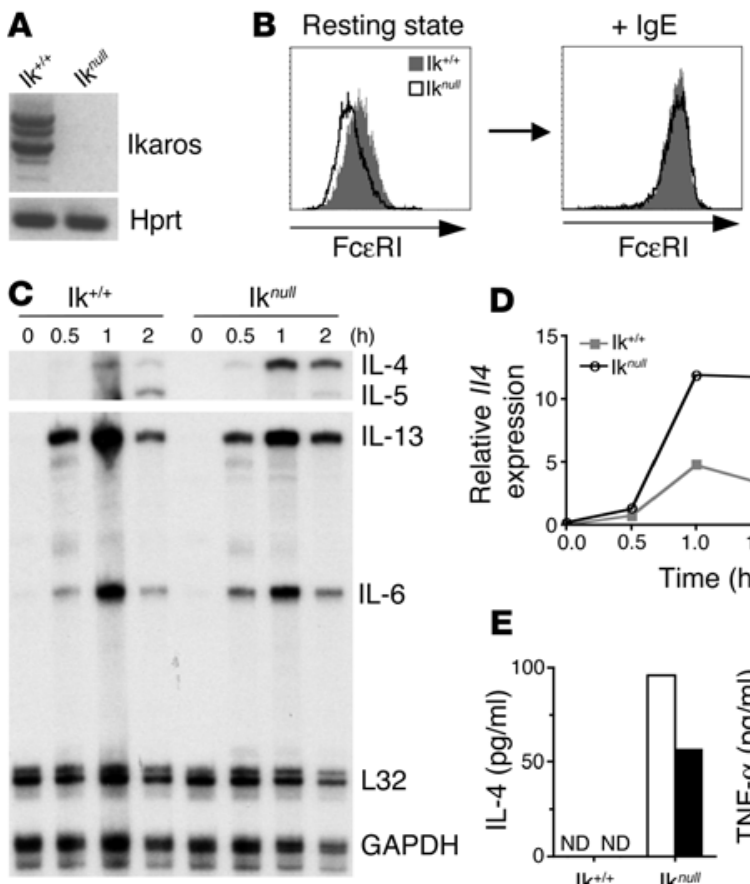

D
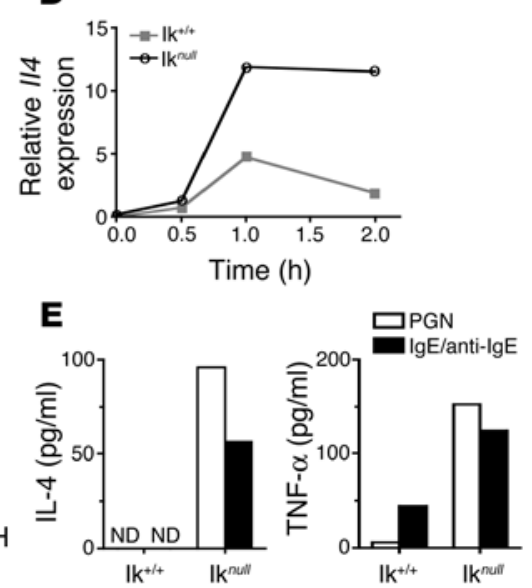

$\mathbf{F}$

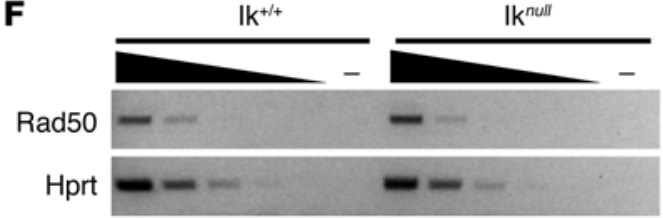

\section{Figure 6}

Ikaros suppresses //4 transcription in MCs. (A) Resting BMMC express a number of Ikaros isoforms. Total RNA was analyzed by RT-PCR for the expression of Ikaros using primers specific for exons 1 and 7 . Expression of the housekeeping gene Hprt was included as a positive control. Absence of Ikaros mRNA expression in Ikaros ${ }^{\text {null }}$ BMMCs was also confirmed. (B) Surface expression of FcERI on BMMCs derived from Ikaros ${ }^{+/+}$and Ikaros ${ }^{\text {null }}$ mice. Cells were left untreated or were cultured with monomeric $\operatorname{lgE}(5 \mu \mathrm{g} / \mathrm{ml})$ overnight. FceRl expression was analyzed by flow cytometry. ( $\mathbf{C}$ and D) Ikaros ${ }^{+/+}$and Ikaros ${ }^{\text {null }}$ BMMCs were stimulated by FcERI cross-linking, and cytokine mRNA expression was analyzed by ribonuclease protection assay. IL-4 mRNA expression was quantified as in Figure 2C. Data are representative of 4 independently derived BMMC cultures. (E) Cytokine secretion by activated lkaros ${ }^{+/+}$and Ikaros ${ }^{\text {null }}$ BMMCs measured 22 hours after activation with peptidoglycan or FceRI crosslinking (IgE/anti-lgE). Mean of 2 independent experiments is shown. ND, not detected. (F) Rad50 and Hprt mRNA expression was analyzed from resting $\mathrm{Ikaros}^{+/+}$and Ikaros ${ }^{\text {null }}$ BMMCs by semiquantitative RT-PCR.

progenitors. Further accessibility leading to a strong $\mathrm{Il} 4$ transcriptional response may be mediated by GATA1.

The observed variability in chromatin accessibility between strains may be inherent to very early MC precursors and/or result from variations in signals downstream of IL-3 and SCF during in vivo and/or in vitro differentiation. Whereas signals delivered by IL-4 and antigens instruct locus opening in Th2 cells, MC growth factors IL-3 and SCF likely work in parallel fashion to affect remodeling during differentiation. Of note, both

Our data correlating chromatin accessibility with IL-4 expression potential in MCs reveals a possible parallel to mechanisms governing the regulation of this gene in Th cells. Several studies have documented that transcription from the Il4 locus in Th2 cells is a stochastic process whereby the probability of expression from either allele is associated with the degree of chromatin accessibility $(27,51,52)$. It is hypothesized that this process allows for a more flexible means of regulation and adds an additional level of control to cytokine gene expression. In Th cells, CNS-1 and $\mathrm{V}_{\mathrm{A}} \mathrm{E}$ are the currently defined targets of probabilistic control, making them key determinants in regulating Il4 expression potential (27). These same sites appeared to influence differential potential for MC Il4 expression. It will be of interest to determine whether there is variability at the single-cell level within MCs of a particular genetic background, as in Th2 cells (51).

Based on our data, we propose a model whereby the integration of GATA and Ikaros signals, which can exert opposing effects on chromatin conformation, are responsible for distinct levels of accessibility at the Th2 locus (Figure 8 ). There are several possible scenarios. For example, Ikaros may be associated with relevant Il4 cis-regulatory elements in early myeloid-lymphoid progenitors and silence expression in these cells. During MC development, lineagespecific transcription factors such as GATA family members function as "pioneering" proteins to decompact the condensed chromatin and promote a cell lineage-specific gene expression profile (53). The extent of GATA pioneering activity may be dependent on relative expression levels or activation states. Thus GATA2 may act as a pioneering factor for initial opening of the Il4 locus in early MC
GATA1 and GATA2 have been implicated as essential transactivators downstream of IL-3 signaling (54). Continued association of Ikaros with relevant elements may allow for a more adaptable locus that can readily alter chromatin accessibility depending on the cells' local microenvironment. This model does not purport that the binding of Ikaros or GATA at individual sites alone can control relative accessibility. Rather, it is the sum of interactions at multiple Th2 locus elements that is likely to dictate the high versus low expression potential. Other possible mechanisms contributing to genetically determined variability in accessibility at this locus could include differences in specific binding patterns of these and other factors, altered ratios of Ikaros to GATA binding, or expression of unique Ikaros isoforms that have distinct functional capabilities. It is also predicted that the interplay between the actions of GATA3 and Ikaros may influence chromatin accessibility in naive and differentiating CD4 T cells, leading to the extremes in expression potential in Th $1 / 2$ cells.

Using a MC knock-in model, we also demonstrated that MCs were required for an optimal antigen-specific IFN- $\gamma$ response by CD4 T cells. More importantly, MC-derived IL-4 was a critical cytokine for this response, supporting the idea that the variable MC IL-4 response in B6 versus Balb/c mice is physiologically relevant. We propose that in certain immune settings, the relatively high IL-4 response of $\mathrm{B} 6 \mathrm{MCs}$ leads to the Th1-dominated responses characteristic of this strain. Conversely, Balb/c MCs do not express sufficient IL-4 to drive a strong Th1 response and default to the Th2 pathway. While this interpretation appears somewhat paradoxical with respect to the conventional Th1/Th2 cell paradigm, these 
A
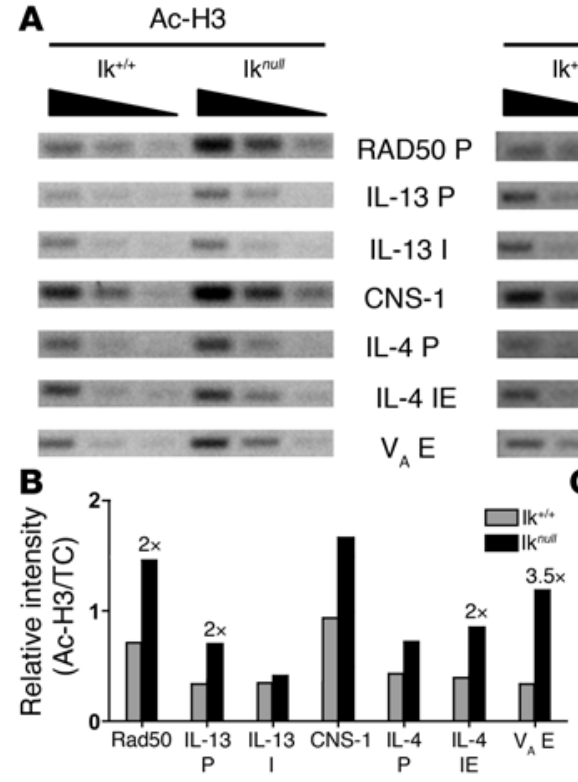

IL-13 P

IL-13।

CNS-1

IL-4 P

IL-4 IE
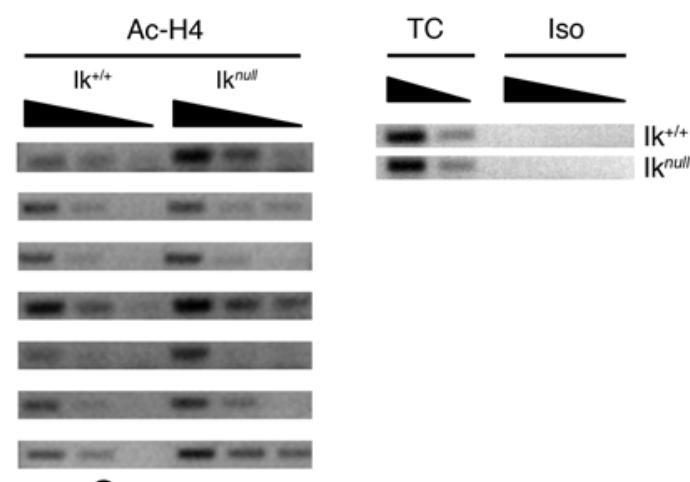

C

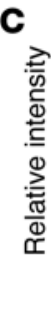

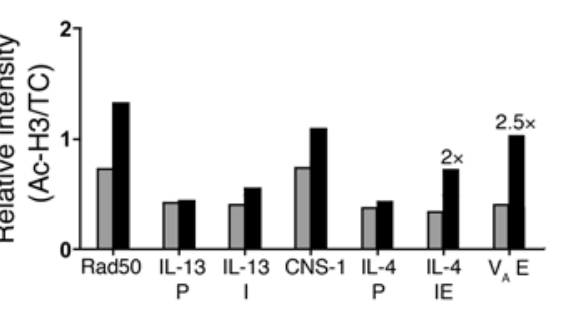

\section{Figure 7}

Ikaros regulates chromatin accessibility of the Th2 locus. (A) Th2 locus accessibility in Ikaros null BMMC was assessed by $\mathrm{H} 3$ and $\mathrm{H} 4$ acetylation using ChIP as in Figure 4. Representative data for total chromatin input and isotype control is shown (CNS-1 primer set). Three-fold dilutions of IP samples were performed, whereas total chromatin input was analyzed using 1:10 and 1:50 dilutions. Data are representative of 3 assays using 2 independently derived MC cultures. (B and C) Relative acetylation of histones $\mathrm{H} 3(\mathbf{B})$ and $\mathrm{H} 4$ (C) were calculated as the ratio of IP template to total chromatin input. Relative intensities at least 2-fold greater than that of $\mathrm{kkaros}^{+/+}$samples are shown above the bars. data highlight the importance of the spatial and temporal expression of IL-4 in the event of a response in vivo. They also support a model proposed by Biedermann et al., who demonstrated that the duration of IL-4 treatment during L. major infection of Balb/c mice directs the character of the Th response (2). When present only during DC maturation, IL-4 induces an IL-12-producing DC1 phenotype promoting Th1 differentiation. If IL- 4 is administered for longer periods and is present during $\mathrm{T}$ cell priming in the secondary lymphoid organs, a typical Th2-dominated response is generated. The ability of MCs to express IL-4 in response to a variety of stimuli and their close proximity to DCs in many tissues including the skin make them likely candidates to modulate DC function in inflammatory settings (55).

There is some indirect evidence to support the idea that genes regulating IL-4 expression potential and chromatin accessibility influence disease heterogeneity. Genes controlling susceptibility to and IL-4 expression during L. major infection map to a number of loci, including L. major response 6 ( $L m r 6$ ). Located at the near end of chromosome 11, Ikaros is found within Lmr6 and is a candi- date susceptibility gene $(56,57)$. Our identification of Ikaros as a gene controlling Th2 cytokine production strengthens these findings. Teuscher and colleagues have also implicated this region in EAE disease severity (58). Further investigations using mice with cell-specific targeted deletions of Ikaros may yield a better understanding of the role of Ikaros in these disease settings.

In summary, although the role of Ikaros is well defined in hematopoietic cell development, little is known about its function in the regulation of genes expressed during immune responses. Our finding that Ikaros was intricately involved in regulation of the Th2 locus in MCs reveals what we believe to be a novel and important role for Ikaros in regulating genes of mature effector cells. Our data also expand our current understanding of the molecular basis for genetically influenced heterogeneity in immune responses and reinforce the idea that MCs are critical regulatory/amplifying components of type 1 immune responses. Such information is critical for refining strategies for changing the class of an immune response, particularly in the setting of vaccine development.

\section{Figure 8}

GATA and lkaros collaborate at the Th2 locus to govern //4 expression potential. (A) Model proposing that Ikaros and GATA proteins - which can associate with Th2 locus cis-regulatory elements concurrently - competitively remodel the local histone state via the recruitment of HDAC or histone acetyltransferase (HAT) activity, respectively. (B) The combination of Ikaros and GATA binding to multiple sites within the Th2 locus, in addition to each factors' inherent ability to recruit suppressive (e.g., HDAC) versus activating (e.g., HAT) complexes, sets the potential for high, intermediate, or low I/4 expression in MCs derived from genetically disparate strains. In Th2 cells, relatively high GATA3 expression leads to efficient opening of the Th2 locus, resulting in a permissive chromatin state.

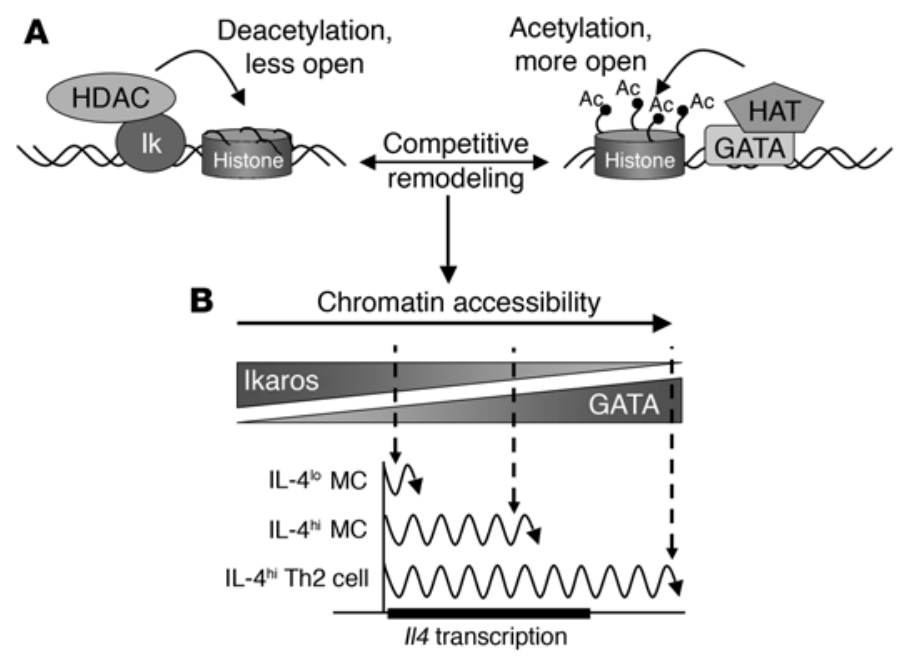




\section{Methods}

Mice. Balb/cJ, SJL/J, B6, and B6-Il tm INnt/J female mice (5-6 weeks old) were purchased from The Jackson Laboratory. Female MC-deficient W/W mice and their congenic littermate controls (WBB6-F1/J-Kit ${ }^{+}-\mathrm{Kit}^{+}$) were obtained at 3 to 5 weeks of age. Ikaros ${ }^{\text {null }}$ mice $(B 6 \times$ Sv129) were originally generated by K. Georgopoulos (Massachusetts General Hospital, Charlestown, Massachusetts, USA) (37). Mice were housed in the animal care facilities at Emory University and Northwestern University. All animal experiments were approved by the Institutional Animal Care and Use Committee at Emory University and the Animal Care and Use Committee at Northwestern University.

Generation of BMMCs and Th2 cells. Bone marrow was harvested from 5- to 6-week-old mice and differentiated with recombinant IL-3 (rIL-3; $5 \mathrm{ng} / \mathrm{ml}$ ) and SCF $(12.5 \mathrm{ng} / \mathrm{ml})$ in complete RPMI (15\% heat-inactivated FBS, $2 \mathrm{mM}$ glutamine, $1 \%$ penicillin-streptomycin, $1 \mathrm{mM}$ sodium pyruvate, and $50 \mu \mathrm{M}$ $2-\beta-\mathrm{ME})$. At $6-8$ weeks of culture, these populations were $>95 \% \mathrm{MCs}$ as assessed by c-kit and FceRI expression and toluidine blue staining. Th2 cells were differentiated in vitro with plate-bound anti-CD28 and antiTCR $\beta$ as previously described (44).

For optimal cytokine expression, MCs were cultured at $5 \times 10^{5}$ cells $/ \mathrm{ml}$ in $5 \mathrm{ng} / \mathrm{ml} \mathrm{rIL-3} \mathrm{(termed} \mathrm{day} 0$ ) and stimulated on day 3 . FceRI cross-linking was accomplished using a previously described mode of IgE/anti-IgE activation (59). RNA was obtained using RNA-STAT60 (Tel-Test Inc.). For activation via TLR agonists, cells were suspended at $5 \times 10^{5}$ cells $/ \mathrm{ml}$ in complete rIL-3-containing RPMI on day 3 and stimulated with $50 \mathrm{ng} / \mathrm{ml}$ LPS or $100 \mu \mathrm{g} / \mathrm{ml}$ sonicated peptidoglycan (Sigma-Aldrich) or heat-killed M. tuberculosis strain H37RA (BD Diagnostics). Th2 cells were activated for 24 hours by plate-bound anti-TCR $\beta(0.5 \mu \mathrm{g} / \mathrm{ml})$. For cytokine protein analysis, activated BMMCs were cultured at $2 \times 10^{6}$ cells/well in $200 \mu \mathrm{l} \mathrm{com-}$ plete DMEM supplemented with IL-3. Culture supernatants were collected at 22 hours, and cytokine levels were assayed using LiquiChip Workstation (Qiagen) as previously described (22).

Ribonuclease protection assays. Assays were performed per the manufacturer's instructions (RiboQuant Multi-Probe RNase Protection Assay System; $\mathrm{BD}$ Biosciences). Gels were visualized by autoradiography and/or quantified via phosphorimaging and ImageQuant software (GE HealthCare).

ChIP. Analysis of histone $\mathrm{H} 3$ and $\mathrm{H} 4$ acetylation and in vivo binding of GATA1/2 and Ikaros was performed using ChIP per the manufacturer's instructions (Upstate USA Inc.) and as previously described (29) using resting BMMCs. A polyclonal $\mathrm{Ab}$ to Ikaros ( $\mathrm{H}-100)$ was obtained from Santa Cruz Biotechnologies Inc. Immunoprecipitates were resuspended in $50 \mu \mathrm{l}$ of $\mathrm{H}_{2} \mathrm{O}$, and serial 3-fold dilutions of $1 \mu \mathrm{l}$ template were used for each reaction. Sequences of primers used in PCR were as follows: Rad50 promoter forward, 5'-GTGCGAGCTTACGGTTGCTGGCTCGC-3'; Rad50 promoter reverse, 5'-AACCTCAAGGCGGAAGCTGGGCAAG-3'; IL-13 promoter forward, 5'-TCCAAACCATGCATTGCTTTGGTG-3'; IL-13 promoter reverse, 5'-TTTGTTGGGCATTATCTGAAAACC-3'; IL-13 intron forward, 5'-GTGAGTAGCACACACAGCCCCTCC- ${ }^{\prime}$; IL-13 intron reverse, 5'-TGATAAACAGTGGTCGCCACTCC-3'; CNS-1 forward, 5'-TGATTTCTCGGCAGCCAGGGAGGGCC-3'; CNS-1 reverse, 5'-GGTGCCTGCGT-
CACCTCTGACCACAC-3'; IL-4 promoter forward, 5'-ACTCATTTTCCCTTGGTTTCAGC-3'; IL-4 promoter reverse, $5^{\prime}$-GATTTTTGTCGCATCCGTGG-3'; IL-4 intron forward, $5^{\prime}$-TCTGCTTGGACATCTCTCTTCCC-3'; IL-4 intron reverse, 5'-ACCACCCCACAGGTCTTTGTTC-3'; $\mathrm{V}_{\mathrm{A}} \mathrm{E}$ forward, 5'-CAGGTCCTGATGCCACCTTAGTAAG-3'; $\mathrm{V}_{\mathrm{A}}$ E reverse, 5' -TTTAAATCCAGATTCTGCATAGAATGG- $3^{\prime}$. PCR conditions were $95^{\circ} \mathrm{C}$ denaturation, $60^{\circ} \mathrm{C}$ annealing, and $72^{\circ} \mathrm{C}$ extension for $30-36$ cycles. PCR products were visualized with ethidium bromide on a $1.5 \%$ agarose gel.

RT-PCR. RNA was obtained from resting MCs as described above. RNA was reverse transcribed using oligo-dT and SuperScript II. The following primers, complementary to exon 1 and exon 7 sequences, were used to amplify Ikaros isoforms: Ik forward, $5^{\prime}$-GATAGATCTATGGATGTCGATGAGGGTCAAGAC-3'; Ik reverse, 5'-GATGAATTCTTAGCTCAGGTGGTAACGATGCTC-3' (60); Hprt forward, 5'-GTTGGATACAGGCCAGACTTTGTTG-3'; Hprt reverse, 5'-GAGGGTAGGCTGGCCTATAGGCT-3'. TLR2 and TLR4, amplified for 35 cycles, were used as previously described (61).

Reconstitution and disease induction. Four-week-old W/Wv mice were reconstituted with $4 \times 10^{6} \mathrm{BMMCs}$ by i.v. transfer. Selective reconstitution of the MC compartment was confirmed by hematocrit, histology, and/or flow cytometry at > 8 weeks after transfer (Supplemental Figure 1; available online with this article; doi:10.1172/JCI27227DS1; and data not shown). EAE was induced by s.c. injection of $300 \mu \mathrm{g} \mathrm{MOG}_{35-55}$ emulsified in $5 \mathrm{mg} / \mathrm{ml} \mathrm{CFA} \mathrm{and} 250 \mathrm{ng}$ pertussis toxin i.p. Mice were scored daily for paralysis as previously described (22).

Intracellular cytokine staining and flow cytometry. Preparation of CNSinfiltrating lymphocytes and in vitro restimulation of splenocytes with $\mathrm{MOG}_{35-55}$ peptide was performed as described previously (22). Intracellular cytokine expression for IFN- $\gamma$ expression was assayed using the Cytofix/ Cytoperm Kit (BD Biosciences) and analyzed by flow cytometry.

Statistics. Statistical analysis was performed using GraphPad Prism software (version 4.0a). Repeated-measures ANOVA followed by Dunnett's multiple-comparison post-test was used for comparison of mean clinical scores in EAE experiments. Intracellular cytokine expression was analyzed by unpaired Student's $t$ test. $P$ values less than 0.05 were considered to be statistically significant.

\section{Acknowledgments}

This work was supported by NIH grant R01A047992 and grant RG3104 from the National Multiple Sclerosis Society (to M.A. Brown).

Received for publication October 26, 2005, and accepted in revised form February 28, 2006.

Address correspondence to: Melissa A. Brown, Department of Microbiology-Immunology, Northwestern University Feinberg School of Medicine, Tarry Medical Research Building 6-758, mail code S213, 320 East Superior Street, Chicago, Illinois 606113010, USA. Phone: (312) 503-0108; Fax: (312) 503-4839; E-mail: m-brown12@northwestern.edu.
1. Weiss, D.L., and Brown, M.A. 2001. Regulation of IL-4 production in mast cells: a paradigm for cell-type-specific gene expression. Immunol. Rev. 179:35-47.

2. Biedermann, T., et al. 2001. IL-4 instructs TH1 responses and resistance to Leishmania major in susceptible BALB/c mice. Nat. Immunol. 2:1054-1060.

3. Min, B., et al. 2004. Basophils produce IL-4 and accumulate in tissues after infection with a Th2inducing parasite. J. Exp. Med. 200:507-517.

4. Launois, P., et al. 2002. Rapid IL-4 production by Leishmania homolog of mammalian RACK1-reac- tive CD4(+) T cells in resistant mice treated once with anti-IL-12 or -IFN-gamma antibodies at the onset of infection with Leishmania major instructs Th2 cell development, resulting in nonhealing lesions. J. Immunol. 168:4628-4635.

5. Brown, M.A., et al. 1987. B cell stimulatory factor-1/ interleukin- 4 mRNA is expressed by normal and transformed mast cells. Cell. 50:809-818.

6. Noben-Trauth, N., Hu-Li, J., and Paul, W.E. 2002. IL-4 secreted from individual naive CD4+ T cells acts in an autocrine manner to induce Th2 differentiation. Eur. J. Immunol. 32:1428-1433.
7. Brown, M.A., and Hural, J. 1997. Functions of IL-4 and control of its expression. Crit. Rev. Immunol. 17:1-32.

8. Ansel, K.M., Lee, D.U., and Rao, A. 2003. An epigenetic view of helper $\mathrm{T}$ cell differentiation. Nat. Immunol. 4:616-623.

9. Ansel, K.M., et al. 2004. Deletion of a conserved Il4 silencer impairs $\mathrm{T}$ helper type 1 -mediated immunity. Nat. Immunol. 5:1251-1259.

10. Lee, D.U., Agarwal, S., and Rao, A. 2002. Th2 lineage commitment and efficient IL-4 production involves extended demethylation of the IL-4 gene. 
Immunity. 16:649-660.

11. Solymar, D.C., Agarwal, S., Bassing, C.H., Alt, F.W. and Rao, A. 2002. A 3' enhancer in the IL-4 gene regulates cytokine production by Th2 cells and mast cells. Immunity. 17:41-50.

12. Lee, G.R., Spilianakis, C.G., and Flavell, R.A. 2005 Hypersensitive site 7 of the TH2 locus control region is essential for expressing $\mathrm{TH} 2$ cytokine genes and for long-range intrachromosomal interactions. Nat. Immunol. 6:42-48.

13. Mohrs, M., et al. 2001. Deletion of a coordinate regulator of type 2 cytokine expression in mice. Nat. Immunol. 2:842-847.

14. Fields, P.E., Lee, G.R., Kim, S.T., Bartsevich, V.V., and Flavell, R.A. 2004. Th2-specific chromatin remodeling and enhancer activity in the th2 cytokine locus control region. Immunity. 21:865-876.

15. Grogan, J.L., et al. 2001. Early transcription and silencing of cytokine genes underlie polarization of T helper cell subsets. Immunity. 14:205-215.

16. Grogan, J.L., et al. 2003. Basal chromatin modification at the IL-4 gene in helper T cells. J. Immunol. 171:6672-6679.

17. Avni, O., et al. 2002. T(H) cell differentiation is accompanied by dynamic changes in histone acetylation of cytokine genes. Nat. Immunol. 3:643-651.

18. Sacks, D., and Noben-Trauth, N. 2002. The immunology of susceptibility and resistance to Leishmania major in mice. Nat. Rev. Immunol. 2:845-858.

19. Hondowicz, B.D., Park, A.Y., Elloso, M.M., and Scott, P. 2000. Maintenance of IL-12-responsive CD4+ T cells during a Th2 response in Leishmania majorinfected mice. Eur. J. Immunol. 30:2007-2014.

20. Robbie-Ryan, M., and Brown, M. 2002. The role of mast cells in allergy and autoimmunity. Curr. Opin. Immunol. 14:728-733.

21. Nakae, S., et al. 2005. Mast cells enhance T cell activation: importance of mast cell-derived TNF. Proc. Natl. Acad. Sci. U. S. A. 102:6467-6472.

22. Gregory, G.D., Robbie-Ryan, M., Secor, V.H., Sabatino, J.J., Jr., and Brown, M.A. 2005. Mast cells are required for optimal autoreactive $\mathrm{T}$ cell responses in a murine model of multiple sclerosis. Eur. J. Immunol. 35:3478-3486.

23. Hochrein, H., et al. 2000. Interleukin (IL)-4 is a major regulatory cytokine governing bioactive IL-12 production by mouse and human dendritic cells. J. Exp. Med. 192:823-833.

24. Yao, Y., Li, W., Kaplan, M.H., and Chang, C.H. 2005. Interleukin (IL)-4 inhibits IL-10 to promote IL-12 production by dendritic cells. J. Exp. Med. 201:1899-1903.

25. Grewal, I.S., et al. 1996. Requirement for CD40 ligand in costimulation induction, $\mathrm{T}$ cell activation, and experimental allergic encephalomyelitis. Science. 273:1864-1867.

26. Yoshimoto, T., Bendelac, A., Hu-Li, J., and Paul, W.E. 1995. Defective IgE production by SJL mice is linked to the absence of CD4+, NK1.1+ T cells that promptly produce interleukin 4. Proc. Natl. Acad. Sci. U. S. A. 92:11931-11934.

27. Guo, L., et al. 2002. In TH2 cells the Il4 gene has a series of accessibility states associated with distinctive probabilities of IL-4 production. Proc. Natl. Acad. Sci. U. S. A. 99:10623-10628.
28. Loots, G.G., et al. 2000. Identification of a coordinate regulator of interleukins 4,13 , and 5 by cross-species sequence comparisons. Science. 288:136-140.

29. Kwan, M., Powell, D.R., Nachman, T.Y., and Brown, M.A. 2005. An intron GATA-binding site regulates chromatin accessibility and is essential for IL-4 gene expression in mast cells. Eur. J. Immunol. 35:1267-1274

30. Henkel, G., et al. 1992. A DNase I-hypersensitive site in the second intron of the murine IL-4 gene defines a mast cell-specific enhancer. J. Immunol. 149:3239-3246.

31. Hural, J.A., Kwan, M., Henkel, G., Hock, M.B., and Brown, M.A. 2000. An intron transcriptional enhancer element regulates IL-4 gene locus accessibility in mast cells. J. Immunol. 165:3239-3249.

32. Sabbattini, P., et al. 2001. Binding of Ikaros to the lambda5 promoter silences transcription through a mechanism that does not require heterochromatin formation. EMBO J. 20:2812-2822.

33. Harker, N., et al. 2002. The CD8alpha gene locus is regulated by the Ikaros family of proteins. Mol. Cell. 10:1403-1415.

34. Asai, K., et al. 2001. Regulation of mast cell survival by IgE. Immunity. 14:791-800.

35. Galli, S.J., Nakae, S., and Tsai, M. 2005. Mast cells in the development of adaptive immune responses. Nat. Immunol. 6:135-142.

36. Gregory, G.D., and Brown, M.A. 2005. Mast cells in allergy and autoimmunity: implications for adaptive immunity. In Mast cells: methods and protocols. G. Krishnaswamy and D.S. Chi, editors. Humana Press Inc. Totowa, New Jersey, USA. 35-50.

37. Georgopoulos, K., et al. 1994. The Ikaros gene is required for the development of all lymphoid lineages. Cell. 79:143-156.

38. Migliaccio, A.R., et al. 2003. GATA-1 as a regulator of mast cell differentiation revealed by the phenotype of the GATA-1low mouse mutant. J. Exp. Med. 197:281-296.

39. Tsai, F.Y., and Orkin, S.H. 1997. Transcription factor GATA-2 is required for proliferation/survival of early hematopoietic cells and mast cell formation, but not for erythroid and myeloid terminal differentiation. Blood. 89:3636-3643.

40. Lee, H.J., et al. 2000. GATA-3 induces T helper cell type 2 (Th2) cytokine expression and chromatin remodeling in committed Th1 cells. J. Exp. Med. 192:105-115.

41. Yamashita, M., et al. 2004. Essential role of GATA3 for the maintenance of type 2 helper $\mathrm{T}$ (Th2) cytokine production and chromatin remodeling at the Th2 cytokine gene loci. J. Biol. Chem. 279:26983-26990.

42. Lee, G.R., Fields, P.E., and Flavell, R.A. 2001. Regulation of IL-4 gene expression by distal regulatory elements and GATA-3 at the chromatin level Immunity. 14:447-459.

43. Monticelli, S., and Rao, A. 2002. NFAT1 and NFAT2 are positive regulators of IL-4 gene transcription. Eur. J. Immunol. 32:2971-2978.

44. Agarwal, S., Avni, O., and Rao, A. 2000. Cell-typerestricted binding of the transcription factor NFAT to a distal IL-4 enhancer in vivo. Immunity. 12:643-652
45. Hutchins, A.S., et al. 2002. Gene silencing quantitatively controls the function of a developmental trans-activator. Mol. Cell. 10:81-91.

46. Makar, K.W., et al. 2003. Active recruitment of DNA methyltransferases regulates interleukin 4 in thymocytes and T cells. Nat. Immunol. 4:1183-1190.

47. Harigae, H., et al. 1998. Differential roles of GATA-1 and GATA- 2 in growth and differentiation of mast cells. Genes Cells. 3:39-50.

48. Trinh, L.A., et al. 2001. Down-regulation of TDT transcription in $\mathrm{CD} 4(+) \mathrm{CD} 8(+)$ thymocytes by Ikaros proteins in direct competition with an Ets activator. Genes Dev. 15:1817-1832.

49. Cobb, B.S., et al. 2000. Targeting of Ikaros to pericentromeric heterochromatin by direct DNA binding. Genes Dev. 14:2146-2160.

50. Kim, J., et al. 1999. Ikaros DNA-binding proteins direct formation of chromatin remodeling complexes in lymphocytes. Immunity. 10:345-355.

51. Guo, L., Hu-Li, J., and Paul, W.E. 2005. Probabilistic regulation in TH2 cells accounts for monoallelic expression of IL-4 and IL-13. Immunity. 23:89-99.

52. Guo, L., Hu-Li, J., and Paul, W.E. 2004. Probabilistic regulation of IL-4 production in Th2 cells: accessibility at the Il4 locus. Immunity. 20:193-203.

53. Cirillo, L.A., et al. 2002. Opening of compacted chromatin by early developmental transcription factors HNF3 (FoxA) and GATA-4. Mol. Cell. 9:279-289.

54. Yu, Y.L., Chiang, Y.J., and Yen, J.J. 2002. GATA factors are essential for transcription of the survival gene E4bp4 and the viability response of interleukin-3 in Ba/F3 hematopoietic cells. J. Biol. Chem. 277:27144-27153.

55. Jawdat, D.M., Albert, E.J., Rowden, G., Haidl, I.D., and Marshall, J.S. 2004. IgE-mediated mast cell activation induces Langerhans cell migration in vivo. J. Immunol. 173:5275-5282.

56. Lipoldova, M., et al. 2000. Susceptibility to Leishmania major infection in mice: multiple loci and heterogeneity of immunopathological phenotypes. Genes Immun. 1:200-206.

57. Beebe, A.M., Mauze, S., Schork, N.J., and Coffman, R.L. 1997. Serial backcross mapping of multiple loci associated with resistance to Leishmania major in mice. Immunity. 6:551-557.

58. Butterfield, R.J., et al. 1998. New genetic loci that control susceptibility and symptoms of experimental allergic encephalomyelitis in inbred mice. J. Immunol. 161:1860-1867.

59. Hock, M.B., and Brown, M.A. 2003. Nuclear factor of activated T cells 2 transactivation in mast cells: a novel isoform-specific transactivation domain confers unique FcepsilonRI responsiveness. J. Biol. Chem. 278:26695-26703.

60. Hahm, K., et al. 1994. The lymphoid transcription factor LyF-1 is encoded by specific, alternatively spliced mRNAs derived from the Ikaros gene. Mol. Cell. Biol. 14:7111-7123.

61. Supajatura, V., et al. 2002. Differential responses of mast cell Toll-like receptors 2 and 4 in allergy and innate immunity. J. Clin. Invest. 109:1351-1359. doi:10.1172/JCI200214704.

62. Heinemeyer, T., et al. 1998. Databases on transcriptional regulation: TRANSFAC, TRRD and COMPEL. Nucleic Acids Res. 26:362-367. 